\title{
Effects of Three Different Stimulations (Acupuncture, Moxibustion, Acupuncture Plus Moxibustion) of BL.67 Acupoint at Small Toe on Fetal Behavior of Breech Presentation
}

\author{
Isabella Neri, Viviana De Pace, Paolo Venturini and Fabio Facchinetti \\ Mother-Infant Department, University of Modena e Reggio Emilia \\ Via del Pozzo 71, 41100, Modena, Italy
}

\begin{abstract}
The aim of the study was to evaluate cardiovascular effects and fetal behavior during moxibustion, acupuncture or acupuncture plus moxibustion applied on the BL.67 acupoint of women (beside the outer corner of the 5th toenail) in fetal breech presentation. During the acupoint stimulation (20 min, two times a week), the women were submitted to computerized non-stress test. Fourteen cases were treated by both acupuncture and moxibustion, 15 cases by moxibustion and 10 cases by acupuncture. In $56 \%$ of cases, fetal position was converted from breech position to cephalic one; the success share was $80 \%$ for moxibustion, $28 \%$ for acupuncture, $57 \%$ for acupuncture plus moxibustion; the conversion, on average, was achieved after 3 sessions. Statistical analysis indicated that acupuncture plus moxibustion was able to reduce fetal heart rate during the application of stimuli while acupuncture and moxibustion separately did not affect such parameter. Moreover, moxibustion and acupuncture with moxibustion reduced fetal movements while acupuncture only appears ineffective. The present study suggests that fetal movements were reduced by both acupuncture plus moxibustion and moxibustion and that fetal heart rate was reduced just by acupuncture plus moxibustion. The mechanisms leading the effect on fetal heart rate and fetal movements remain to be clarified. Even though further studies are needed, such preliminar report mainly investigated the impact of different stimula on the BL.67 acupoint. Unfortunately these small series of data do not allow us to draw any conclusion about the effectiveness of the different treatments.
\end{abstract}

Keywords: Acupuncture; Moxibustion; Acupuncture Point BL.67 Point; Breech Presentation; Computerized Non-stress Test.

Correspondence to: Dr. Isabella Neri, Via del Pozzo 71, 41100 Modena, Italy. Tel: (+39) 59-422-2664, Fax: (+39) 59-422-4394, E-mail: neri.isabella@unimo.it 


\section{Introduction}

In case of fetal malposition, Chinese traditional medicine purposes the stimulation of the acupoint 67 of the bladder meridian (BL.67), which is at the lateral corner of the nail bed of the 5th toenail, with acupuncture, moxibustion or acupuncture plus moxibustion (in order to strengthen the stimuli produced by the simple acupuncture application), (Cooperative Research Group of Moxibustion Version of Jangxi Province, 1980 and 1984; Cardini et al., 1998). In a randomized, controlled study performed by us, thanks to the acupuncture plus moxibustion, we were able to converte $58 \%$ of cases of breech position to cephalic one (Neri et al., 2004), whereas no reports are available on the impact of moxibustion or acupuncture, separately, on the BL.67 acupoint in western population.

In order to clarify the mechanisms leading to cephalic version, we have observed the effects of acupuncture plus moxibustion in respect to minimal acupuncture on computerized non-stress test that checks and records fetal wellness. Such findings showed that the BL.67 acupoint stimulation was able to induce changes in cardiovascular and behavioral fetal variables, namely fetal movements (Neri et al., 2003). The aim of the present study is to compare the effect of acupuncture plus moxibustion, moxibustion or acupuncture on fetal behavior.

\section{Materials and Methods}

We considered for the study 45 primigravid women referred to the Acupuncture Clinic of the Obstetric Department because carrying breech presented fetuses. Women had maternal age ranged between 20 and 40 years, gestational age ranged between 33 and 36 weeks of gestation and fetuses all in frank position. Exclusion criteria were: previous uterine surgery, twin pregnancies, low level of amniotic fluid, maternal renal or cardiac disease, fetal malformation or chromosome abnormality.

Two women were refused for acupuncture because of low compliance toward the method and two women were excluded for previous uterine surgery (1 case) or presence of low aminiotic fluid (1 case). Finally, 41 women were randomised to receive acupoint stimulation, but the analysis was performed on 39 cases because of 2 cases ( 1 in acupuncture plus moxibustion and 1 in moxibustion group) have not reached the baseline cardiotocographic criteria requested to define an active period as above described. Acupuncture plus moxibustion (14 cases), moxibustion ( 15 cases) or acupuncture (10 cases) was applied on the acupoint BL.67 (beside the outer corner of the 5th toenail, Fig. 1) bilaterally for $20 \mathrm{~min}$.

Acupoint stimulations were performed twice a week in order to strengthen the stimuli produced by the simple acupuncture application. During the stimulation of acupoint BL.67, women were submitted to computerized non stress test through a Hewlett-Packard 8040 cardio-tocograph (Fig. 2) equipped with a wide range external ultrasound transducer and a trans-abdominal toco-dynamometer (HP-2CTG, Hewlett-Packard Division, Italy). A cardio-tocograph is able to check and record fetal wellness analyzing several variables such as fetal heart rate, fetal movements (thanks to the ultrasound transducer) and uterine 


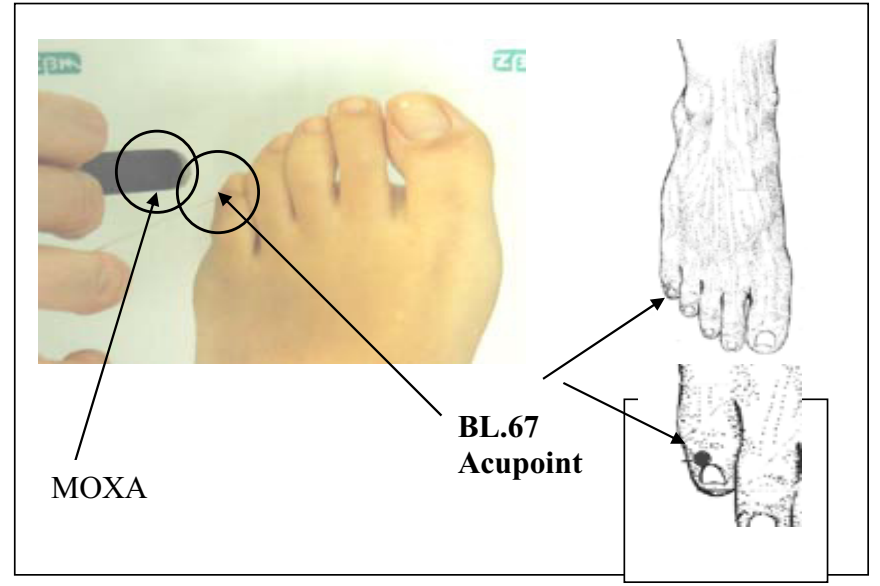

Figure 1. BL.67 acupoint.

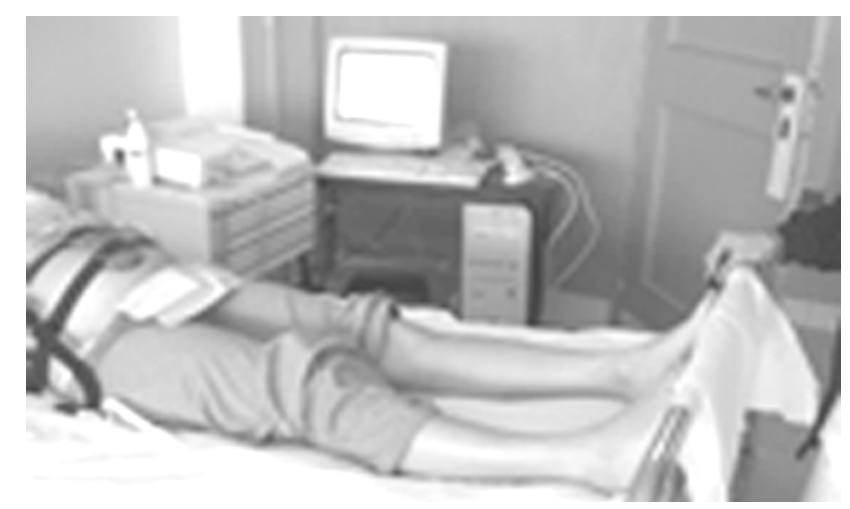

Figure 2. A computerized cardio-tocograph analyzing fetal behavior during a session of stimulation of the acupoint BL.67 with moxibustion.

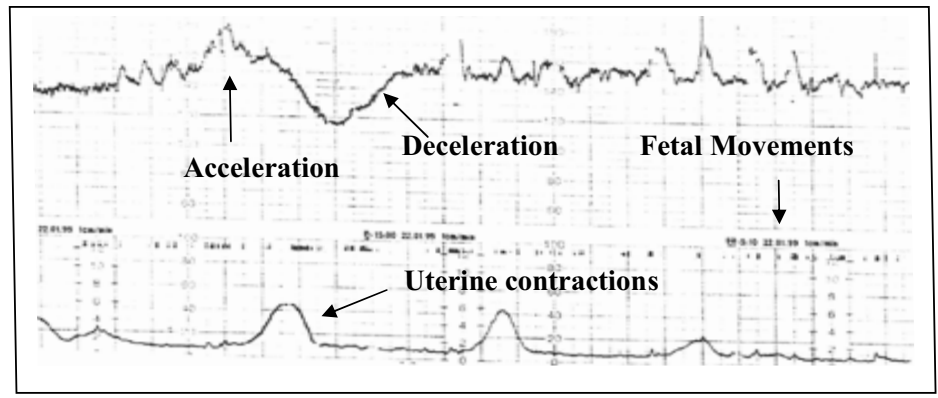

Figure 3. Variables analyzed by a cardio-tocograph are able to check fetal wellness. 
contractions (thanks to the trans-abdominal toco-dynamometer) (Fig. 3). All the recordings were performed at the same time (10:00 a.m.-12:00 p.m.) in a comfortable, semi-recumbent position. Computerized non-stress test started $20 \mathrm{~min}$ before the stimuli application, continued during treatment and lasted for further $20 \mathrm{~min}$ after the end of the stimulation.

The following variables of computerized non-stress test, evaluating fetal wellness, were considered: baseline fetal heart rate, number of large accelerations defined as fetal heart rate variations above the baseline of amplitude greater than 15 beats/min for at least $15 \mathrm{sec}$, number of small accelerations defined as fetal heart rate variations above the baseline of amplitude greater than 10 beats/min for at least $12.5 \mathrm{sec}$, long term variability evaluated with the long term irregularity index, short term variability evaluated every $60 \mathrm{sec}$ and defined as average of successive $2.5 \mathrm{sec}$ pulse intervals epochal differences, number of decelerations as number of fetal heart rate variations below the baseline of amplitude greater than 20 beats/min for at least $30 \mathrm{sec}$ or greater than 10 beats/min for at least $60 \mathrm{sec}$. Fetal movements were recorded by a trans-abdominal tocodynamometer. The stimulus was applied during an "active" period. As published elsewhere (Arduini et al., 1993), we analyzed by cardiotocograph $5 \mathrm{~min}$ epochs which were considered "active" if the following criteria were satisfied: variability of fetal heart rate greater than 10 beats/ min, presence of accelerations of fetal heart rate and presence of fetal movements. Epochs were considered inadequate when the fetal heart rate signal was lost or disturbed and did lend itself to optimal analysis. Data are reported as mean \pm standard deviation. Multiple analyses of variance for repeated measures were applied. This kind of statistical analysis is able to evaluate within-group changes of non-stress test variables. Post-hoc comparison with paired t-test was also done. A p-value and an F value less than 0.05 was considered as statistically different as commonly accepted (Gaddis, 1998).

\section{Results}

No differences were observed in acupuncture plus moxibustion, moxibustion and acupuncture groups for maternal age $(32.8 \pm 4.8$ vs $34.1 \pm 5.6$ vs $33.4 \pm 7.4$ years $)$ and gestational age at the inclusion ( $34.1 \pm 0.9$ vs $34.1 \pm 0.7$ vs $35.2 \pm 7.2$ weeks). Computerized non-stress test variables recorded and analyzed before the beginning of stimulation were similar between groups as shown in Table 1 .

Concerning the acupoint stimulation, multiple analyses of variance for repeated measures indicate that the fetal heart rate changes during the treatment application $(\mathrm{F}=$ $0.480, p=0.05)$. Indeed the t-test showed that acupuncture plus moxibustion was able to reduce fetal heart rate whereas acupuncture and moxibustion separately do not affect such parameter (Fig. 4). The other non-stress test variables remain unaffected by treatments.

However, multiple analysis of variance for repeated measures also indicates that fetal movements change during stimuli application $(\mathrm{F}=0.377, \mathrm{p}=0.05)$. The $\mathrm{t}$-test showed that both acupuncture plus moxibustion and moxibustion applications reduce fetal movements whereas no effect were shown by applying acupuncture (Fig. 5), The effects on fetal behavior of these methods remain to be explained considering that fetal movements and fetal heart rate regulation is not yet clearly known. 
Table 1. The Baseline Values of Non-stress Test

\begin{tabular}{|c|c|c|c|c|}
\hline & $\begin{array}{c}\text { Acupuncture + Moxibustion } \\
\left(n^{\circ} 14\right)\end{array}$ & $\begin{array}{c}\text { Moxibustion } \\
\left(\mathbf{n}^{\circ} 15\right)\end{array}$ & $\begin{array}{l}\text { Acupuncture } \\
\quad\left(\mathbf{n}^{\circ} \mathbf{1 0}\right)\end{array}$ & $\mathrm{p}<0.05$ \\
\hline $\begin{array}{c}\text { Fetal Heart Rate } \\
\text { (bpm) }\end{array}$ & $\begin{array}{c}145.8 \pm 12.6 \\
(129.4-169.6)\end{array}$ & $\begin{array}{c}146.4 \pm 10.6 \\
(131.2-163.6)\end{array}$ & $\begin{array}{l}139.1 \pm 8.3 \\
(126-148)\end{array}$ & n. s. \\
\hline $\begin{array}{l}\text { Short Term Variability } \\
\text { (bpm) }\end{array}$ & $\begin{array}{c}6.6 \pm 2 \\
(4.3-10.7)\end{array}$ & $\begin{array}{c}6.0 \pm 1.6 \\
(4.7-11.5)\end{array}$ & $\begin{array}{l}6.7 \pm 1.6 \\
(5.1-9.1)\end{array}$ & n. s. \\
\hline $\begin{array}{l}\text { Long Term Variability } \\
\text { (bpm) }\end{array}$ & $\begin{array}{l}26.7 \pm 11.1 \\
(13.1-47.7)\end{array}$ & $\begin{array}{l}25.3 \pm 10.11 \\
(13.1-47.3)\end{array}$ & $\begin{array}{l}20.9 \pm 7.5 \\
(12.9-34)\end{array}$ & n. s. \\
\hline $\begin{array}{c}\text { Large Accelerations } \\
\qquad\left(\mathbf{n}^{\circ}\right)\end{array}$ & $\begin{array}{c}4.1 \pm 2.3 \\
(0-8)\end{array}$ & $\begin{array}{c}3.8 \pm 3.2 \\
(1-8)\end{array}$ & $\begin{array}{l}5.1 \pm 2.6 \\
(2-8)\end{array}$ & n. s. \\
\hline $\begin{array}{c}\text { Small Accelerations } \\
\qquad\left(\mathbf{n}^{\circ}\right)\end{array}$ & $\begin{array}{c}1.9 \pm 1.8 \\
(0-4)\end{array}$ & $\begin{array}{l}3 \pm 3 \\
(0-8)\end{array}$ & $\begin{array}{l}2.1 \pm 2.2 \\
\quad(0-6)\end{array}$ & n. s. \\
\hline
\end{tabular}

n. $\mathrm{s} .=$ Not significant, $\mathrm{n}^{\circ}=$ number of cases.

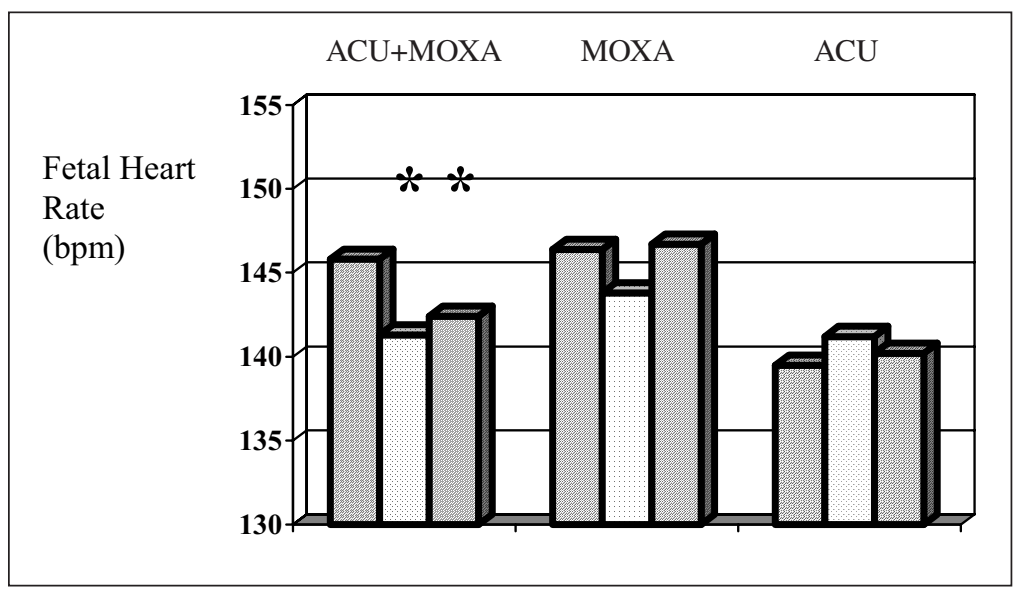

Figure 4. The changes of fetal heart rate before, during and after acupoint stimulation.

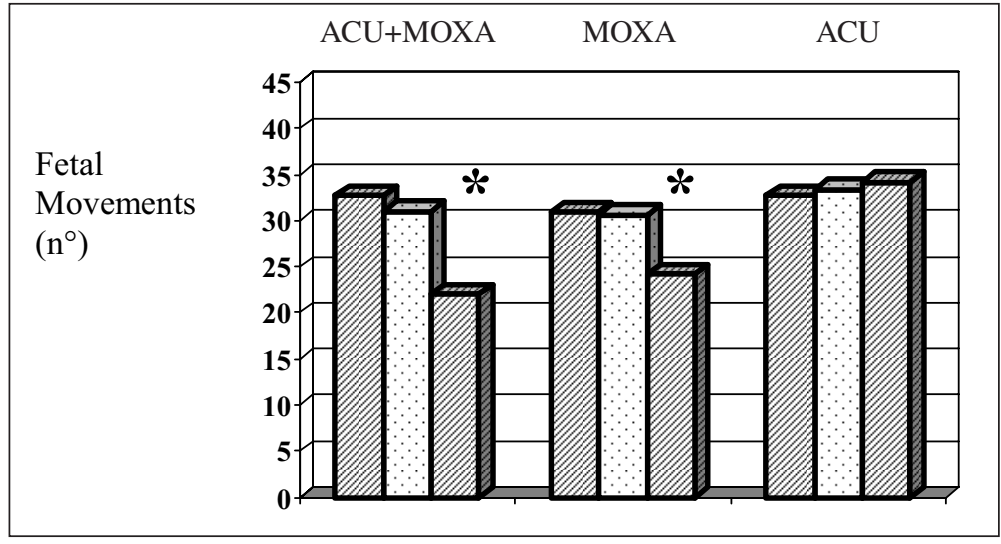

Figure 5. The changes of fetal movements before, during and after acupoint stimulation. 
Table 2. Success Share Described for Kind of Stimulation

\begin{tabular}{lccc}
\hline & $\begin{array}{c}\text { Acupuncture + Moxibustion } \\
\left(\mathbf{n}^{\circ} \mathbf{1 4}\right)\end{array}$ & $\begin{array}{c}\text { Moxibustion } \\
\left(\mathbf{n}^{\circ} \mathbf{1 5}\right)\end{array}$ & $\begin{array}{c}\text { Acupuncture } \\
\left(\mathbf{n}^{\circ} \mathbf{1 0}\right)\end{array}$ \\
\hline Success Share (\%) & $8 / 14$ & $12 / 15$ & $2 / 10$ \\
& $57 \%)$ & $(80 \%)$ & $(28 \%)$ \\
\hline
\end{tabular}

$\mathrm{n}^{\circ}=$ Number of cases.

In $56 \%$ of cases, fetal position was converted from breech position to the cephalic position; the share of success of the single kind of stimulation is reported in Table 2.

\section{Discussion}

Application of acupuncture plus moxibustion was able to reduce fetal heart rate and fetal movements as previously demonstrated by us (Neri et al., 2003), where moxibustion without needle insertion affects just fetal movements and acupuncture without moxibustion do not affect fetal behavior. Interestingly, the changes of fetal heart rate occurred just during treatment and the changes of fetal movements occurred after the application. The probable difference could be related to the different mechanisms involved in the regulation of fetal heart rate and fetal movements.

The small series of data does not allow us to make a hypothesis about the effectiveness of the single treatments; the fact that single acupuncture does not affect fetal variables, does not mean that this treatment is ineffective.

Such effects on fetal behavior remain to be explained considering that the mechanism of fetal movements and fetal heart rate regulation is not yet clearly established. It has been reported that naloxone, antagonist of endogenous oppioid peptides, increased fetal body movements, heart rate accelerations and breathing movements and that endogenous oppioid exert a tonic inhibition of fetal movements (Arduini et al., 1987). Considering acupuncture application was able to stimulate central and peripheral endorphins, the effect of acupuncture on fetal behavior can be explained (Sjoelund et al., 1977).

A reduction of fetal heart rate was described by Arduini et al. (1988) after feto-maternal adrenal gland suppression by triamcinolone. Many processes affect fetal heart rate, but the most studied interference has been in endocrine control. Indeed, it has been reported that fetal heart rate rhythm is affected by the suppression of the feto-maternal adrenal gland accompanied by maternal cortisol changes (Bennet et al., 1999). It is possible that acupuncture affects feto-maternal cortisol production.

Penn and Steer (1994) reported that breech fetuses showed significant differences in the number of behavioral state transitions with respect to those observed in cephalic presentation. Perhaps acupuncture could play a role in obtaining a cephalic version throughout the modulation of the relative amount of quiescent and active states.

Even though further studies are needed in order to understand the mechanism leading to acupuncture effect, such preliminary report represents an extent to clarify the impact of 
BL.67 acupoint stimulation on fetal behavior probably mediated by endogenous opioid systems or changes in cortisol production.

\section{References}

Arduini, D., G. Rizzo, G. Piana, A. Bonalumi, P. Brambilla and C. Romanini. Computerized analysis of fetal heart rate: I. Description of the System (2CTG). J. Mater. Invest. 3: 159-163, 1993.

Arduini, D., G. Rizzo, S. Dell'Acqua, S. Mancuso and C. Romanini. Effect of naloxone on fetal behavior near term. Am. J. Obstet. Gynecol. 156: 474-478, 1987.

Arduini, D., G. Rizzo and C. Parlati. Modifications of ultradian and circadian rhythms of fetal heart rate after feto-maternal adrenal gland suppression: a double blind study. Prenat. Diagn. 8: 479-484, 1988.

Bennet, L., S. Kozuma, H.H. McGarrigle and M.A. Hanson. Temporal changes in fetal cardiovascular, behavioural, metabolic and endocrine responses to maternally administered dexamethasone in the late gestation fetal sheep. Br. J. Obstet. Gynaecol. 106(4): 331-9, 1999.

Cardini, F. and H. Weixin. Moxibustion for correction of breech presentation. A randomized controlled trial. JAMA 1: 1580-1584, 1998.

Cooperative Research Group of Moxibustion Version of Jiangxi Province. Studies of version by moxibustion on Zhiyin points. In: Z. Xiangton (ed.) Research on Acupuncture, Moxibustion and Acupuncture Anesthesia. Science Press, Beijing, China, 1980.

Cooperative Research Group of Moxibustion Version of Jiangxi Province. Further studies on the clinical effect and the mechanism of version by moxibustion. Abstracts of the Second National Symposium on Acupuncture, Moxibustion and Acupuncture Anesthesia. Beijing, China, 1984.

Gaddis, M.L. Statistical methodology: IV. Analysis of variance, analysis of covariance, and multivariate analysis of variance. Acad. Emerg. Med. 5: 258-265, 1998.

Neri, I., G. Aiola, G. Contu, G. Allais, F. Facchinetti and C. Benedetto. Acupuncture plus moxibustion to resolve breech presentation: a randomized controlled study. J. Mater. Med. 15: 247-252, 2004.

Neri, I., M. Fazzio, S. Menghini, A. Volpe and F. Facchinetti. Non-stress test changes during acupuncture plus moxibustion on BL.67 point in breech presentation. J. Soc. Gynecol. Invest. 9: 156-162, 2003.

Penn, Z.J. and P.J. Steer. Breech presentation. In: D.K. James, P.J. Steer, C.P. Weiner and B. Gonik (eds.) High Risk Pregnancy; Management Options. WB Saunders, London, 1994.

Sjoelund, B., L. Terenius and M. Eriksson. Increased cerebrospinal fluid levels of endorphins after electro-acupuncture. Acta Physiol. Scand. 100: 382-385, 1977. 
Copyright of American Journal of Chinese Medicine is the property of World Scientific Publishing Company and its content may not be copied or emailed to multiple sites or posted to a listserv without the copyright holder's express written permission. However, users may print, download, or email articles for individual use. 